\title{
Heuristic Approaches for Two-Dimensional Bin Packing Problems in Building Material Transportations
}

\author{
Pavee Siriruk \\ Suranaree University of Technology \\ 111 University Ave, Nakhon Ratchasima, Thailand \\ pavee@g.sut.ac.th
}

\begin{abstract}
In the shipping department of building material stores, a batch of objects of various sizes are loaded and shipped as many as possible in a rectangular area of truck beds. The objective of building material stores is to use the least number of trucks possible to satisfy customer demands. Two types of items, square pallets and rectangular products, are considered in this paper. The heuristic approach with the aid of decision trees to minimize transportation costs for the firm. Numerical examples with two sizes of square pallets and one size rectangular products are given. The results show that an algorithm can find the optimal solution with the largest useful space and all items are loaded on truck bed.
\end{abstract}

Keywords: Two-Dimensional Bin Packing Problems, Heuristic Methods, Layout Problem.

\section{Introduction}

In the Two-Dimensional Bin Packing Problem (2BP), it is required to allocate a set of rectangular items to a larger rectangular bin, and the objective is to allocate all the items to minimize the number of bins. There are many applications related to 2BP. In the shipping department of building material stores, a batch of objects of various sizes are loaded and shipped as many as possible in a rectangular area of truck beds. Small and large items are placed together on a square shaped pallet before loading on the truck beds, while larger items with square or rectangular shape, which cannot put on pallets, are put on the truck beds directly. The process of placing pallets and items on the truck beds is the same as allocating a set of rectangular items to a larger rectangular bin. The pallets and large items are assumed to have rectangular shapes. Truck beds are considered as rectangular bin. This process plays an important role in transportation costs. If a store can reduce the number of trucks used in transportation, the store could reduce the transportation costs.

The objective of building material stores is to use the least number of trucks possible to satisfy customer demands. In other words, the pallets and objects are placed on the truck bed so that the area of the smallest rectangle bounding all the objects is minimized. In the final layout, it is desired that there should be no overlap of any objects while they are placed as close to each other as possible.

There are many algorithms related to $2 \mathrm{BP}$ such as Scheithauer [4] considered the problem of optimal packing of small pieces within larger pallets. The paper investigates equivalence and dominance of packing patterns which leads to an efficient branch and bound algorithm. The objective is to find a subset of the pieces which can be packed in a feasible manner within the pallet and which minimizes the unused area. A set of pieces is feasibly packed, if they do not overlap each other. Martello and Vigo [7] presented the exact solution of the two-dimensional finite bin packing problem. The lower bound is analysed and the worst-case performance is determined. Lower bounds, used within a branch and bound algorithm for the exact solution of the problem, is proposed. The numerical example, involving up to 120 pieces, is considered and solved to show the effectiveness of the proposed approach. Jakobs [6] introduced genetic algorithms for solving the orthogonal packing problem. The orthogonal packing problem is calculation of the orthogonal packing pattern with minimal height. These problems frequently occur in steel and textile industries. The aim is to maximize the use of contiguous remainder of the board and piece of cloth. The genetic algorithm is modified and improved by combination with deterministic methods. The researcher also points out a direction to improve in the future research. Lodi, Martello, and Vigo [1] investigated twodimensional bin packing problems by introducing a new heuristic algorithm and a unified tabu search approach that is adapted 
to a specific problem by simply changing the heuristic used to explore the neighborhood. The results showed that tabu search generally improves the initial deterministic solution produced by the inner heuristic, which is effective in all cases. Moreover, they also presented approximation algorithms for the oriented two-dimensional bin packing problem. [2] The algorithm is a simple deterministic approximation algorithm, which is used in the initialization of a tabu search approach. The tabu search algorithm is presented and analyzed its average performance through extensive computational experiments. The results show an effective behavior of the proposed tabu search. Gea and Hae [5] proposed genetic algorithm for two-dimensional packing problems. The algorithm is applicable to not only convex shaped objects, but can also handle any type of concave and complex shaped objects including objects with holes. This approach is improved over previous genetic algorithms in introducing a new concept of a two-dimensional genetic chromosome. The total layout space of a rectangular bin is divided into a finite number of cells for mapping it into 2D genetic algorithm chromosome. The results show that several goods layouts are obtained. Zhang, Deng, and Kang [3] introduced a hybrid heuristic algorithm for the two-dimensional rectangular packing problem. The divine-and-conquer approach is used. The algorithm breaks a problem into subproblems that are similar to the original problem but smaller in size. The subproblem is solved recursively and combined these solutions to create a solution to the original problem. The results shown that hybrid heuristic algorithm outperformed other two heuristics in percent of unpacked area and CPU time. Liu, Chu, and Wang [8] presented a new heuristic algorithm for a class of twodimensional bin-packing problems. In this research, a class of different customer demands are considered such as orientation of items. The heuristic algorithm is developed from dynamic programming idea by aggregating states to avoid the explosion of the number of states. This algorithm has advantage over other algorithms in its flexibility, competitive computation time, and performance with respect to metaheuristics. Two-dimensional bin packing problems are solved using heuristic algorithm. The results showed that this heuristic algorithm can become a general heuristic method to solve a series of set partitioning problems as well as real world situation.

In this paper, we develop the heuristic approach with the aid of decision trees to minimize transportation costs for the firm. For each transportation truck, an attempt is made to place the objects so that the area of the smallest rectangle bounding all the objects is minimized. In the final layout of all trucks, it is desired that there should be no overlap of any items on truck beds, while they are placed as close to each other as possible. The paper is organized as follows. A problem description is presented in section two. In section three, the solution methodology is proposed. The numerical examples and results are shown in section four. In section five, the results of this research are concluded.

\section{Problem Description}

Based on real operations in building material transportations, it is assumed that the store have $k$ trucks available for transportation. Each empty truck bed has length $L$ and width $W$. Given $i$ types of square pallets with the number of $n_{i}$ pallets. Each pallet type $i$ has length $l_{i}$ and width $w_{i}, 1 \leq i \leq n$. Moreover, given $j$ types of large rectangular products with the number of $m_{j}$ products. Each type of product $j$ has length $a_{j}$ and width $b_{j}, 1 \leq j \leq m$. Small items are placed together on a square shaped pallet before loading on the truck beds, while larger items with rectangular shape are put on the truck beds directly. The pallets are assumed to have only square shape, while large items are assumed to have rectangular shapes and can be rotated by 90 degrees before placing on truck beds. One common way of minimizing transportation costs for the building material store is by using the least number of trucks possible. This method should help the enterprises make packing plans. In the final layout, it is desired that there should be no overlap of any objects while they are placed as close to each other as possible.

\section{Solution Methodology}

In this section, the heuristic approach with decision trees are developed. The heuristic approach involves five steps:

1) Pack square pallets on the first row starting from upper left corner of the trucks. If there is no square pallets, go to step 2. Choose the number of square pallets that have the least empty space on the first row as depicted in Figure 1. In other words, choose to pack pallet type $i$ that satisfies the following equation:

$$
\operatorname{Min}_{i}\left\{L-l_{i} \times\left(L \operatorname{div} l_{i}\right)\right\} \quad \text { for } \quad 1 \leq i \leq n
$$


Loading Pallets on the first row

Least empty space

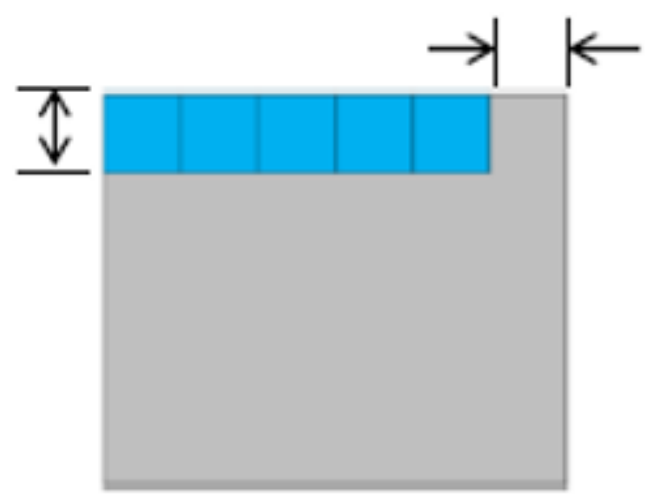

Fig. 1: The method to load pallets for the first row.

2) After the first row of truck bed is filled regardless of height, deploy decision trees to make decision on packing all large rectangular products which can be rotated by 90 degrees. Choose a branch on the decision tree that has the emptiest spaces on truck bed. In other words, the rectangular products are placed as close to each other as possible.

3) Load the rest of items on the truck bed continuing from left to right.

a) If the remaining items are square pallets with the same size, decision tree is not required. Load square pallets on truck bed starting from left to right.

b) If the remaining items are square pallets with different sizes, the larger sized square pallets are loaded on truck bed first starting from left to right.

4) If there is not enough space on truck bed, one more truck is employed and go to step 1.

5) Choose the arrangement that maximize useful spaces on truck bed.

\section{Numerical Examples}

Given that one building material store has 1 truck available for transportation. Each empty truck bed has 2,000 mm in width $(W=2,000)$ and 2,400 $\mathrm{mm}$ in length $(L=2,400)$. There are 2 types of square pallets with the number of 7 pallets for each type. Pallet type 1 has $500 \mathrm{~mm}$ in width and length. In addition, pallet type 2 has $400 \mathrm{~mm}$ in width and length. The data are shown in Table 1.

Table 1: Data for square pallets.

\begin{tabular}{|c|c|c|c|}
\hline$i$ & $n_{i}$ & $w_{i} \times l_{i}(\mathrm{~mm})$ & Nomenclature \\
\hline 1 & 5 & $500 \mathrm{~mm} \times 500 \mathrm{~mm}$ & A \\
\hline 2 & 7 & $400 \mathrm{~mm} \times 400 \mathrm{~mm}$ & D \\
\hline
\end{tabular}

Moreover, given $j$ types of large rectangular products with the number of $m_{j}$ products. Each type of product $j$ has length $a_{j}$ and width $b_{j}, 1 \leq j \leq m$.

There is one type of rectangular products with 7 pieces to deliver. The product has $450 \mathrm{~mm}$ in width and $650 \mathrm{~mm}$ in length. Since the product can be rotated 90 degrees, we can place it in horizontal and vertical orientation. When we place the product in horizontal orientation, it is represented by $B$. In contrast, when the product is placed in vertical orientation, it is represented by $C$. The data are shown in Table 2 . 
Table 2: Data for rectangular products.

\begin{tabular}{|c|c|c|c|}
\hline$j$ & $m_{j}$ & $w_{j} \times l_{j}(\mathrm{~mm})$ & Nomenclature \\
\hline \multirow{2}{*}{1} & \multirow{2}{*}{7} & $450 \mathrm{~mm} \times 650 \mathrm{~mm}$ & $\mathrm{~B}$ \\
\cline { 3 - 4 } & & $650 \mathrm{~mm} \times 450 \mathrm{~mm}$ & $\mathrm{C}$ \\
\hline
\end{tabular}

The algorithm described in section 3 is used to solve numerical example above.

Pack square pallets on the first row of truck bed starting from upper left corner. Using equation 1 above, the results is shown below.

Square pallet type 1 (A) : $2400-500 \times(2400$ div 500) $=400 \mathrm{~mm}$

Square pallet type 2 (D) : $2400-400 \times(2400 \operatorname{div} 400)=0 \mathrm{~mm}$

Choose square pallet that yields the lowest value from equation 1 . Thus, we choose to load square pallet type 2 (D) on a truck bed first. Since truck bed has $2,400 \mathrm{~mm}$ in length, we can only fit 6 of pallet type 2 with no empty space as depicted in Figure 2. There is one pallet type 2 left which will load on truck bed later.

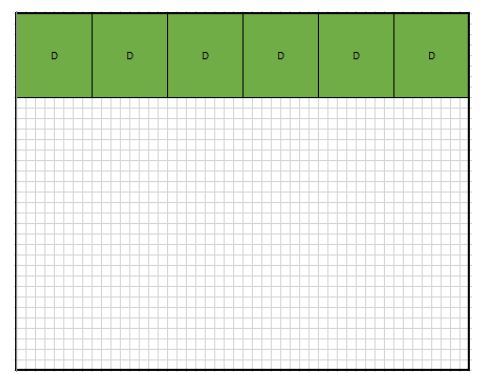

Fig. 2: Load square pallet type 2 on truck bed.

Decision tree is used to list all possibilities on packing all large rectangular products. As 7 pieces of rectangular products, which can be rotated by 90 degrees, are considered, horizontal orientation is denoted by B and vertical orientation is denoted by $\mathrm{C}$. There are 128 possible layouts. The best possible arrangement is B-B-B-C-C-C-C, which has the highest useful space on truck bed. In other words, all items are placed as close to each other as possible. The arrangement is shown in Figure 3. The grey area in Figure 3 is called useful areas which can put more items on truck bed.

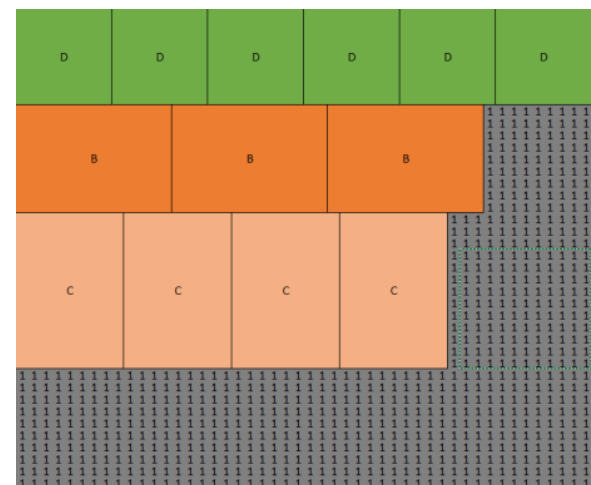

Fig. 3: Finding the best layout using decision trees.

After all rectangular products are loaded on the truck, all square pallet type 1 (A) and square pallet type 2 (D) are loaded on truck bed from left to right. The second truck is not required, since all pallets can be loaded on the truck as depicted in Figure 4 below. The useful space is $382,500 \mathrm{~mm}^{2}$ which is shown in grey area in Figure 4 . 


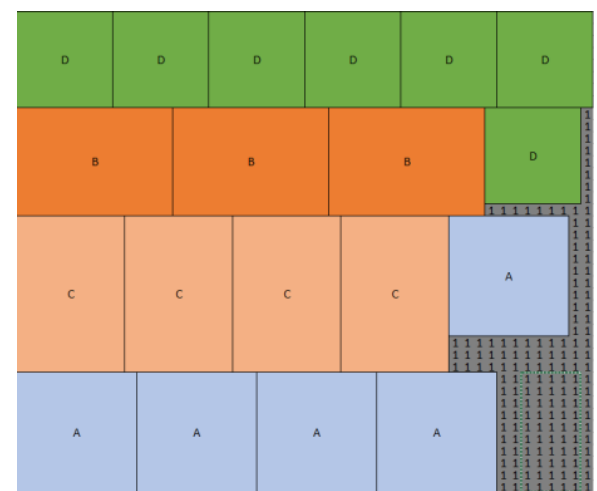

Fig. 4: Finding the best layout with all items on truck bed.

Thus, only one truck is used in the shipment process and all items can be loaded on a truck bed with $382,500 \mathrm{~mm}^{2}$ useful space left.

\section{Conclusions}

In this paper, the heuristic approaches for two-dimensional bin packing problems in building material transportations were proposed. The operation of building material transportations was described. Two types of items, square pallets and rectangular products, were considered. The heuristic approach with decision tree was developed. Numerical examples with two sizes of square pallets and one size rectangular products are given. The results showed that an algorithm can find the optimal solution with the largest useful space and all items are loaded on truck bed. However, the metaheuristic approaches are more efficient in finding solutions which are future research directions.

\section{References}

[1] A. Lodi, S. Martello, and D. Vigo, "Approximation algorithms for the oriented two-dimensional bin packing problem," Eur. J. Oper. Res., vol. 112, no. 1, pp. 158-166, 1999.

[2] A. Lodi, S. Martello, and D. Vigo, "Heuristic and Metaheuristic Approaches for a Class of Two-Dimensional Bin Packing Problems," INFORMS J. Comput., vol. 11, no. 4, pp. 345-357, 1999.

[3] D. Zhang, A. Deng, and Y. Kang, "A Hybrid Heuristic Algorithm for the Rectangular Packing Problem," Int. Conf. Comput. Sci., pp. 783-791, 2005.

[4] G. Scheithauer, "Equivalence and dominance for problems of optimal packing of rectangles," Ric. Oper., vol. 83, no. February, p. 3-34., 1997.

[5] S. J. and H. C. Gea, "Two-Dimensional Packing Problems Using Genetic Algorithms," Eng. Comput., vol. 14, no. 3, pp. 206-213, 1998.

[6] S. Jakobs, "On genetic algorithms for the packing of polygons," Eur. J. Oper. Res., vol. 88, no. 1, pp. 165-181, 1996.

[7] S. Martello and D. Vigo, "Exact Solution of the Two-Dimensional Finite Bin Packing Problem," Manage. Sci., vol. 44, no. April 2015, pp. 388-399, 1998.

[8] Y. Liu, C. Chu, and K. Wang, "A new heuristic algorithm for a class of two-dimensional bin-packing problems," Int. J. Adv. Manuf. Technol., vol. 57, no. 9-12, pp. 1235-1244, 2011. 